\title{
A Kushi-English-Hausa Wordlist
}

\author{
Gian Claudio Batic \\ University of Naples 'L'Orientale'
}

The Kushi language (ISO 639-3 [kuh]; Glottocode: kush1236) is spoken by about 11,000 people in the Kushi 'village area' along the northern foothill of the Chonge-Mona range (Gombe State, Nigeria). Kushi is a Chadic language belonging to the Tangale proper of the Bole-Tangale group (West Chadic).

This basic vocabulary falls within an ongoing documentation project targeting the language and the oral traditions of the Kushi people. The vocabulary is organised into two sections: Kushi-English-Hausa (main section) and English-Kushi (reversal section). Each entry in the main section is organized as follows: <lexeme I part of speech I English gloss I Hausa gloss I (literal transaltion) | (Kushi example I free translation of Kushi example in English I free translation of Kushi example in Hausa)>. Kushi has two contrastive tones, high (H) and low (L), and two contour tones, falling (HL) and rasing (LH). Tones are always marked. If the vowel is long, the tone is marked only on the first vowel (e.g. 6òslì 'North'). Verbs are given in the imperative form. 


\section{Kushi-English-Hausa}

PA

\begin{tabular}{|c|c|}
\hline Pábùrùl & n. okra. ku6ewa. \\
\hline Pàdéelè & n. hail. Kankara. \\
\hline Pàdúnúnò & n. horn. Kaho. \\
\hline Pàdô & n. 1. eye. ido. 2. face. fuska. \\
\hline Pádáy & n. gazelle. barewa. \\
\hline Pàddô & v. eat (meat). ci (nama) \\
\hline Pàdéewè & n. bird. tsuntsu. \\
\hline Pádéyré & $\begin{array}{l}\text { n. scorpion. kunama. fògún Pádénré sting } \\
\text { arbi. }\end{array}$ \\
\hline Páeshàw & n. grain. hatsi, tsaba. \\
\hline Pàgwàgwá & n. duck. agwagwa. \\
\hline Pàkúlà & n. mantis. koki-koki. \\
\hline Pàkùmóm & n. hedgehog. bushiya. \\
\hline Pàládè & $n$. pig. alade. \\
\hline Pàlàw & $n$. leaf. ganye. \\
\hline Pàllágà & $n$. house rat. Gera, kusu. See: shìwrì. \\
\hline Pàlúurà & n. needle. allura. \\
\hline Pàmmò & v. 1. climb up. hawa. 2. ride horse hau doki. \\
\hline Pámít & n. upper stone. dutse na sama. \\
\hline Pànò & v. pay for debt. biya bashi. \\
\hline Pàn & $n$. possessor, owner of. mai. \\
\hline Pàn díyè & n. potter. mai (ginin) tukwane. \\
\hline Pàn gàngày & n. drummer. makadi. \\
\hline \multicolumn{2}{|c|}{$\begin{array}{l}\text { Pàn gèerù téré } n \text {. evening star. zara. Lit: 'person waiting } \\
\text { the moon'. }\end{array}$} \\
\hline Pàn gèy & n. enemy. abokin gaba. \\
\hline Pàn gùnùl & $n$. priest. fada. \\
\hline Pàn kə̀nímmà & n. rainmaker. \\
\hline Pàn kpáanì & n. tanner. majemi. \\
\hline
\end{tabular}

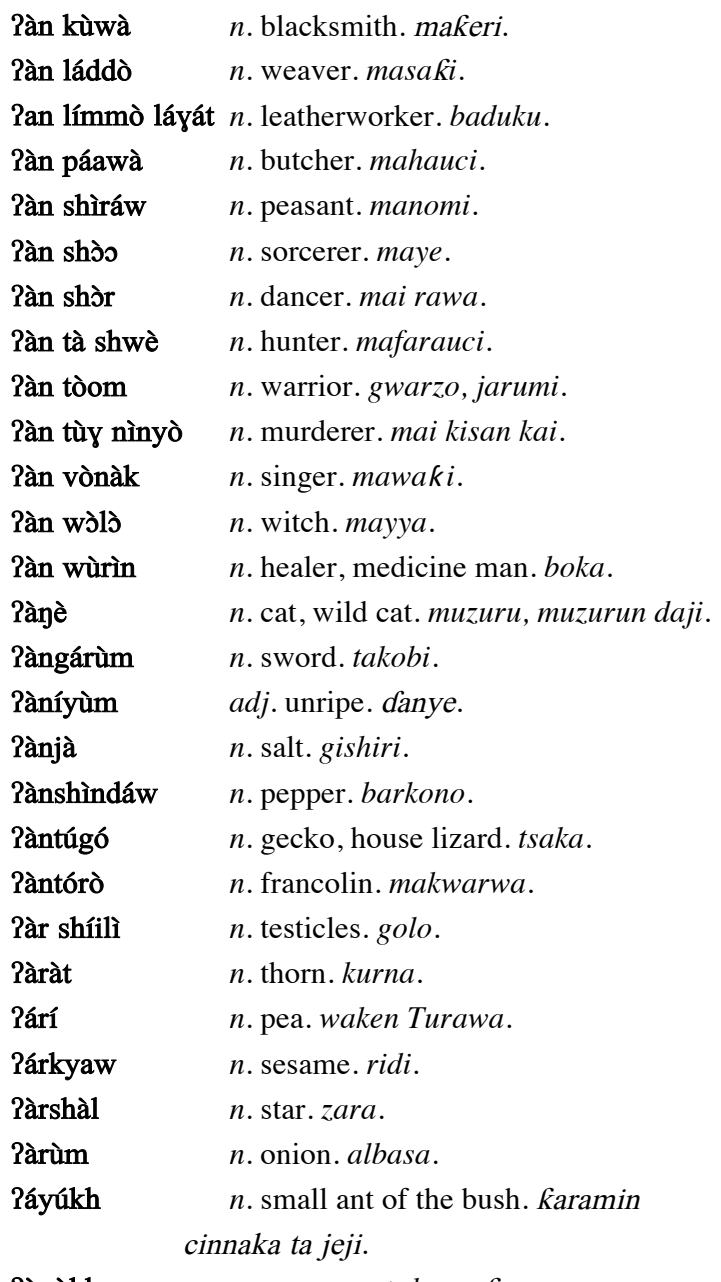

Pàyùkh n. rat. kusa, Gera.

B

$\begin{array}{ll}\text { bèe } & v . \text { shoot. harba. } \\ \text { bànà } & n . \text { antelope. mazo, ragon ruwa, gwanki. } \\ \text { bàrà } & n \text {. trap.tarko. } \\ \text { bèndè } & n \text {. penis sheath. gidan azzakari. } \\ \text { bì } & 1 . n \text {. back. baya.2.prep. behind. (a) baya }(n) . \\ \text { bì bìró } & n \text {. bark of tree. Gawo. Lit: 'behind (of) tree'. } \\ \text { bì gbón } & n \text {. nape (of neck). Keya. } \\ \text { bì shò } & n \text {. footprint. sawun Kafa. }\end{array}$
bìì̀m $\quad n$. heel. diddige.
bìlìr $n$. flower. fure.
bìlìt See: bìlìr.
bìndìgà $n$. gun. bindiga.
bìnnò $\quad n$. guest, stranger. bako.
bìró $\quad n$. tree with no leaves. itace. là bìró stick sanda.
bùkwátì $n$. bucket. bokiti.




$\begin{array}{ll}\text { 6àakh } & n . \text { wing. fiffike. } \\ \text { 6àkà } & n . \text { natron. } \\ \text { 6álá } & n \text {. tiredness. gajiya. } \\ \text { 6án } & n . \text { strength. Karfi. } \\ \text { 6án fok } & n . \text { courage. jaruntaka. } \\ \text { 6ánnì } & n . \text { baobab tree. kuka. } \\ \text { 6ày } & n . \text { dog. kare. } \\ \text { 6ə̀ək } & n . \text { wing(s). fiffike. } \\ \text { 6èrà } & n . \text { facial markings. sasshawa. } \\ \text { 6ìrò } & v . \text { beat. buga. }\end{array}$

$\begin{array}{ll}\text { Gìr(ì) shàw } & n . \text { flail. } \\ \text { 6òy bìró } & n . \text { trunk. gangar jiki. } \\ \text { 6òolì } & n . \text { east. gabas. } \\ \text { 6òròn } & \text { type of millet. irin gero. } \\ \text { 6ùk } & n . \text { soil. kasa. } \\ \text { 6wàarò diyò } & n . \text { divorce. saki. } \\ \text { 6wáré } & n . \text { rattle. caki. } \\ \text { 6wèyù } & n . \text { underworld. } \\ \text { 6wòy } & n . \text { today. yau. }\end{array}$

D

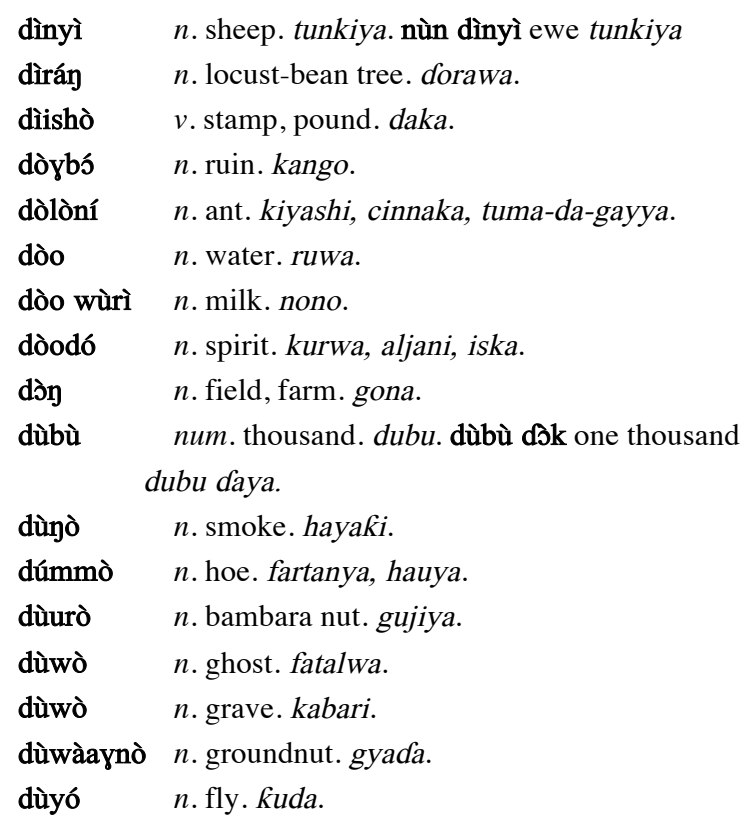

D

$\begin{array}{ll}\text { dák } & n . \text { tongue. harshe. } \\ \text { dáwán } & v . \text { bundle. dami. } \\ \text { dîgù } & v \text {. build. gina. } \\ \text { dîikó } & n . \text { mortar. turmi. } \\ \text { dímím } & n . \text { time. lokaci. } \\ \text { dímmín } & \text { inter. when? yaushe? } \\ \text { dîjò } & v . \text { cook. dafa. } \\ \text { dîyò } & n . \text { marriage. aure. } \\ \text { dîyè } & n . \text { small pot for water. kula. }\end{array}$

\begin{tabular}{|c|c|}
\hline dôybó & n. clay. laya, lumbu. \\
\hline đóylá & $n$. cultivated ground. noma. \\
\hline dok & num. one. daya. \\
\hline dôorò & $n$. pipe. lofe. \\
\hline dúndì & $n$. bottle gourd. buta. \\
\hline dûmmò & v. boil tafasa. \\
\hline dûulò & v. cool. huce. \\
\hline dúulàn & adj. wet. jikakke. \\
\hline dwèy & n. tomorrow. gobe. \\
\hline
\end{tabular}


Pélé $\quad n$. voice. murya.

\begin{tabular}{|c|c|}
\hline fâràgbànày & num. six. shidda. \\
\hline fârlów & num. seven. bakwai. \\
\hline féè & n. chicken. kaza. là féè chick sagara \\
\hline fînk & n. arrow. kibiya. \\
\hline léríyà & n. malaria. zazzabin cizon sauro. \\
\hline fînnè & n. thigh. cinya. \\
\hline fo dán & n. doorway. Kofa. \\
\hline fô dòo & $n$. spring of water. idon ruwa. \\
\hline fo gùn & $n$. anus. dubura. \\
\hline fô kírá & $n$. finger. yatsa. \\
\hline fô lùwàk & n. liver. hanta. \\
\hline fô shə̀ərì & $n$. heart. zuciya. \\
\hline fô shúrùk & n. riverbank. bakin kogi. \\
\hline fô tèerè & $a d v$. there. can. \\
\hline fôdómlì & $n$. sickle (for cutting grass only). lauje. \\
\hline fódd̄ & weed, pluck out. yi noma. \\
\hline dûlò & v. worship. yi bauta, bauta wa. \\
\hline yèràw & num. nine. tara. \\
\hline
\end{tabular}

Pèm n. ring. zobe.

$\mathrm{F}$

fôk $\quad$. 1. mouth, lip. baki, lebe. 2. language. harshe, yare.

fonnò father's brother. kawu, baffa.

fôrà kúnò $\quad n$. escape. tsira, gudu.

forò $n$. forehead. gaba.

fôoró $\quad n$. punishment. horo.

fosshénnì $a d v$. there. can.

fotè $\quad a d v$. here. nan.

foý $\quad n$. black ant. cinnaka.

fûddô $v$. wash clothes. wanke tufafi.

fûgrò $n$. bellows. zugazugi.

fùgùm $n$. blind. makaho.

fúlá $\quad n$. rope. igiya.

fullùm $n$. silk cotton tree (white flowers). rimi.

fûumò $\quad n$. tse-tse fly. Kudan tsando.

fúvùl $n$. foam. kumfa.

fúwàt num. five. biyar. 


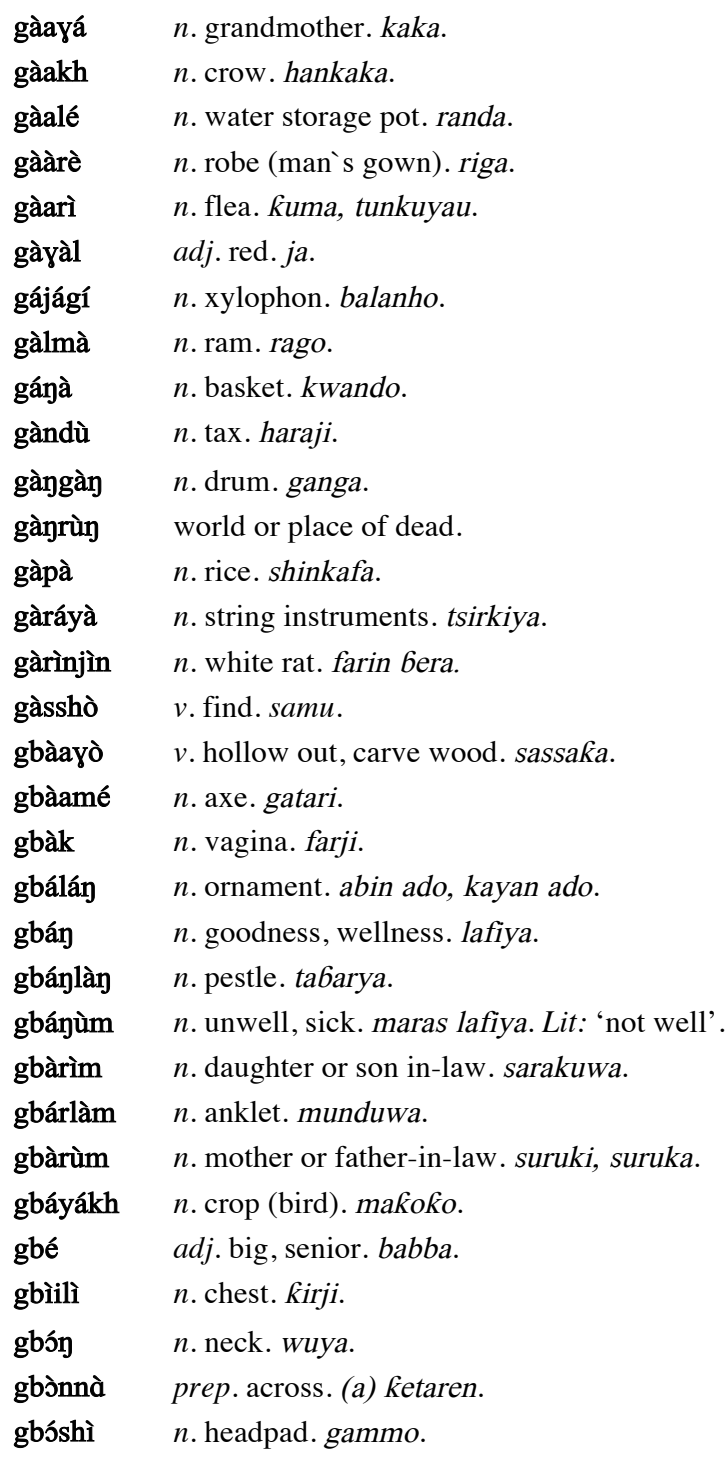

Pìshì fôk

adj. dry, dried. use of verb kafe.

Pìshì shwè $n$. thirst. Kishirwa. Lit: 'dry mouth'.

n. cornstalk. kara.

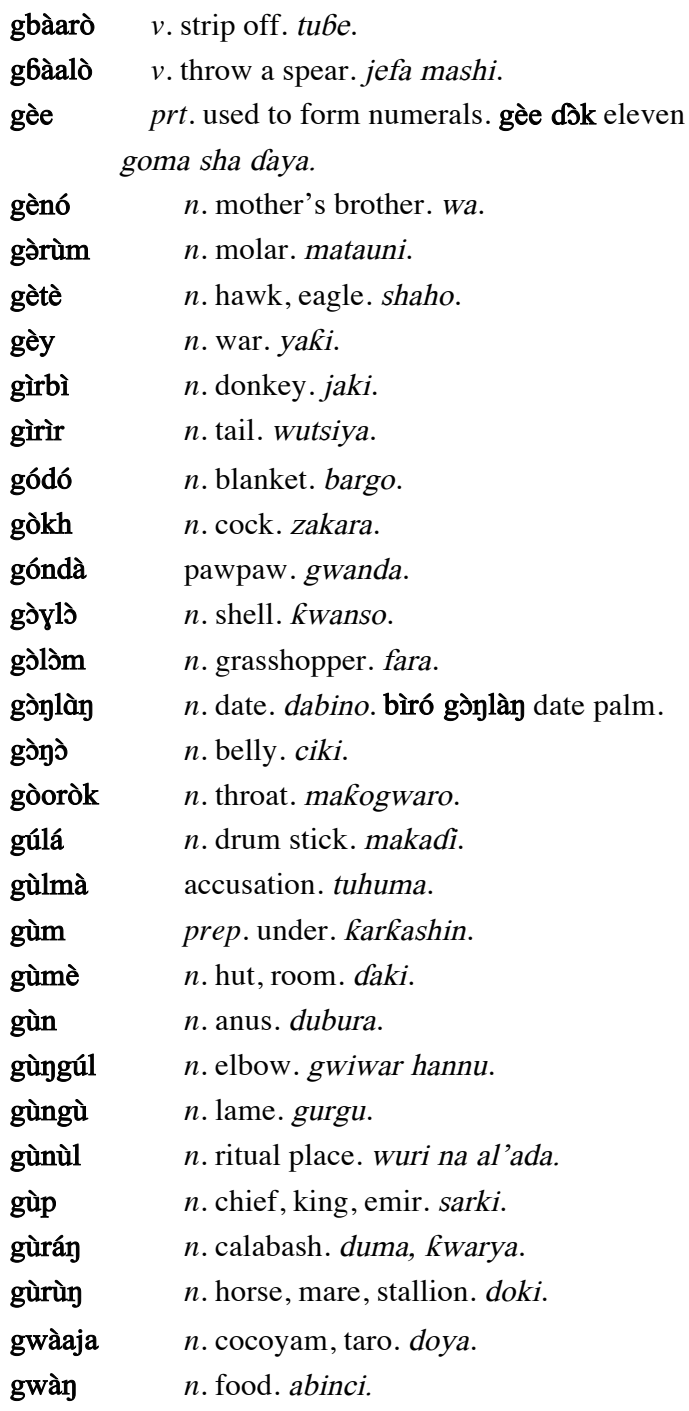

PI

Pìshò $\quad v$. dry up. janye.

Rìshò $\quad v$. grind. Garza, nika. 


\begin{tabular}{|c|c|}
\hline jáylùm & n. cheek. kunci. \\
\hline jàmmó & n. loom. masaka. \\
\hline ján & n. jaw. mummuke. \\
\hline jàyàní & $n$. leopard. damisa. \\
\hline jàrà & $n$. big basket for corn. kwando. \\
\hline jàwéddà & $n$. small knife used for harvesting. \\
\hline jáwít & n. cutlery. wuKa, cokali. \\
\hline jèdè & n. shield. garkuwa. \\
\hline jə̀ə & $n$. bean(s). wake. \\
\hline jèlà & $n$. side. (a) hannu. jèlè jìyaw left side hannun \\
\hline & hagu jèlè témmè right side hannun dama. \\
\hline jéné & n. caterpillar. gizaka. \\
\hline jènè & $n$. dryness, aridity. rani. \\
\hline jènyè & n. crab. Kaguwa. \\
\hline jèrà gòyj̀ & n. ribs. hakarkari. \\
\hline jèrè & $n$. friend, age-mate. aboki. \\
\hline jèshílè & n. trumpet. kakaki. \\
\hline î̂ิ & n. porcupine. beguwa. \\
\hline $\mathrm{iji}$ & $n$. place of burning. \\
\hline jìijí & n. 1. grandfather. kaka. 2. ancestor. mutanen \\
\hline
\end{tabular}

\begin{tabular}{|c|c|}
\hline jìkò & n. rich. mai arziki. \\
\hline jìrà & n. bed. gado. \\
\hline jírí & n. thief. Garawo. \\
\hline jìrìm & n. spirit of a place. iska. \\
\hline jìró & $n$. red monkey. \\
\hline jìwò & $v$. snare. zarge. \\
\hline jìwúr & n. worm. tsutsa. \\
\hline jìyaw & $a d v$. left. hagu. \\
\hline jòd & $n$. filter. mataci. \\
\hline j’̀k & $n$. black wasp. zanzaro. \\
\hline jórró & $n$. locust. fara. \\
\hline jùjákh & n. straw. haki. \\
\hline jùk & $n$. elephant. giwa. \\
\hline jùk & n. chair. kujera. \\
\hline jùnròn & $n$. basket for corn. \\
\hline júr & n. squirrel. kurege. \\
\hline jùwò & $v$. jump. yi tsalle. \\
\hline jwál & $n$. hole. \\
\hline jwál fíră & n. scar, notch. tabo. \\
\hline jwàlé & $n$. cricket. gyare. \\
\hline
\end{tabular}




\begin{tabular}{|c|c|c|c|}
\hline kàawò & v. sow. shuka. & kòdô & n. claw. Kambori. \\
\hline kà dàràn & n. daylight, noon. rana, tsakar rana. & kóyó & n. road. hanya. \\
\hline káasùwà & n. market. kasuwa. & kóyó dàm & n. vein. jijiya. Lit: 'way (of) blood'. \\
\hline kàmmà & n. lion. zaki. & kòlò & n. widow. bazawara. \\
\hline kán & n. granary. rumbu. & kòn & $n$. judge. alkali. \\
\hline kàr kúwò & n. circumcision. kaciya. & kòomò & n. maize. masara. \\
\hline káráráwá & $n$. bell. Karrarawa. & kòshò & $v$. filter. tace. \\
\hline kàsh & n. spear. mashi. & kpàa & n. horn. Kaho. \\
\hline káshò & n. harvest. kaka. & kpèyù & $n$. snake living in water. macijin ruwa. \\
\hline kàshò & $v$. harvest. gira, rora, cira. farta, tona. & & See: kúrét. \\
\hline kàyò & $v$. hoe yi noma. & kpíshì & n. dust cloud. hadarin Kura. \\
\hline kàyò táy & v. cut into pieces. yayyanka. & kpòmù & num. ten. goma. \\
\hline kónó & n. money. kudi. & kpàanò & $v$. tan. jeme. kpáanì \\
\hline kə̀rə̀k & n. border. iyaka. & kpòolś & $n$. yesterday. jiya. \\
\hline kə̀nímmà & n. fetish. juju. & kù & prep. on. a kan. \\
\hline kèlà & n. monitor lizard. damo; guza; tsari. & kù dày & n. 1. sky. sama. 2. heaven. Aljanna. \\
\hline kènè dîyò & n. cowrie. wuri. & kù mínná & n. roof. jinka. Lit: 'head of house'. \\
\hline kènnì & $n$. poor. matalauci. & kùbùk & n. land, ground. Kasa. \\
\hline kérákh & n. mahogany tree. madaci. & kúmá & n. corpse. gawa. \\
\hline kèrè & n. roof of granary. jinkar rumbu. & kúmmí & n. navel. cibiya. \\
\hline kèrèm & n. crocodile. kada. & kúnná & n. dysentery. atuni. \\
\hline kèrò & v. measure. auna, gwada. & kúmó & n. ear. kunne. \\
\hline khà & $v$. wound. yi wa rauni. & kùmùm & n. doubt. shakka. \\
\hline kháa & n. gruel. kunu. & kù péewè & $n$. world. duniya. \\
\hline khàyukh & n. termite. gara, gina, zago. mínná & kúrét & n. snake. maciji. See: rêw, shàmmàm. \\
\hline & shàyukh termite pile suri. & kùurò & n. bitter tomato. gauta. \\
\hline khèeyàw & $n$. white sesame. ridi. & kùurò dذ̀o & type of bitter tomato (not dried). data. \\
\hline khèwnè & $n$. bush cow. saniya. & kùwò & v. lend. ranta, ara wa. \\
\hline khóý & n. track. hanya. & kùwò & $n$. penis. azzakari. \\
\hline khù & n. head. kai. & kùwà & $v$. forge. makera. \\
\hline khùrùwò & $n$. cowry. wuri. & kùwák & n. curse. laPana. \\
\hline kíná & See: kóná. & kúwó & n. debt. bashi. \\
\hline kírá & n. arm, hand. hannu. & kúyám & $n$. hunger. yunwa. \\
\hline kírá bìró & n. branch. reshe. Lit: 'arm (of) tree'. & & \\
\hline kíríyípó & n. dawn, evening. assalatu, asuba, & & \\
\hline
\end{tabular}


L

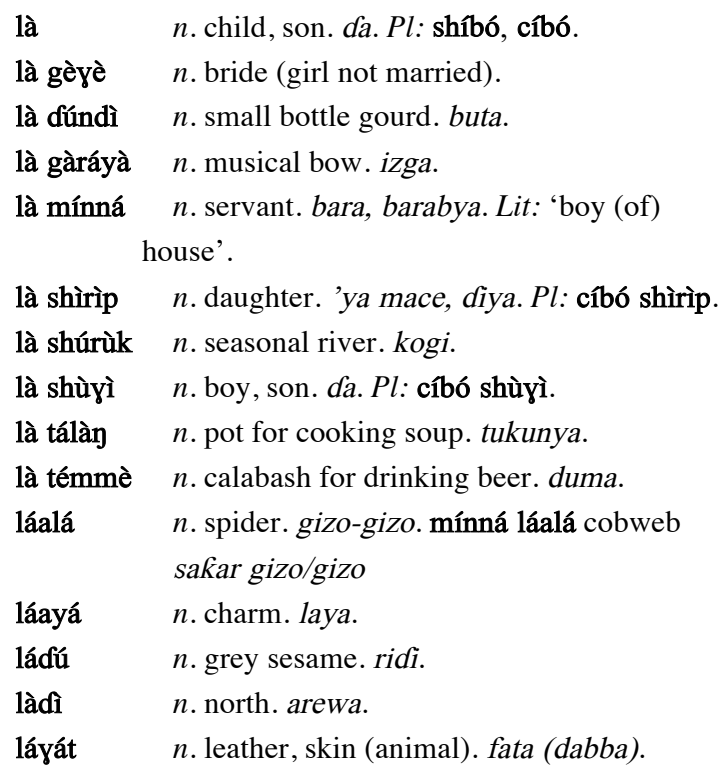

\begin{tabular}{|c|c|}
\hline láyát (shìk) & n. fatar jiki. \\
\hline láját & $n$. outfit of hunter. rigar mafarauci. \\
\hline làkà & n. orphan. maraya. \\
\hline làllò & $v$. ruminate. ci (saniya). \\
\hline làmbú & n. garden. gadina, lamu. \\
\hline lànnà & n. clothes. tufafi. \\
\hline lèeláw & n. cotton. auduga. \\
\hline léyá & n. desire. sha?awa. \\
\hline lèwò & $v$. want, like, love. so. \\
\hline lí & $v \cdot$ put. $s a$. \\
\hline lìlámdè & n. butterfly. malam-buda-mana-littafi. \\
\hline lò̀ & $\begin{array}{l}\text { n. meat. nama. l̀̀(o) tà shwè bush meat naman } \\
\text { daji }\end{array}$ \\
\hline lúrú & n. bag. jaka. \\
\hline lùgnì & $n$. shade. inuwa. \\
\hline lùmmò & $n$. flour. gari. \\
\hline lùmmò 6ùk & $n$. fine sand. rairayi. \\
\hline
\end{tabular}

M

\begin{tabular}{|c|c|}
\hline màlàc & n. rainbow. bakan gizo. \\
\hline màlàc dòo & See: màlàc. \\
\hline màlyò & n. lightning. walkiya. \\
\hline màmmà & n. ant-hill. jiba, suri, shuri. \\
\hline mànlày & n. skull. Kwakwalwa, kwanya. \\
\hline mánná & n. husband. miji. \\
\hline mə̀n & $n$. beer. giya. \\
\hline mə̀n díyè & $n$. beer pot. tulun giya. \\
\hline mè Pàn & $a d v$. many. da yawa. \\
\hline méemé & n. wasp. zanzaro. \\
\hline Mèerò & $v$. make, braid rope. \\
\hline mèrà & n. travel. tafiya. \\
\hline mìdô & n. stirring stick. muciya. \\
\hline mìigù & n. owl. mugiya. \\
\hline millán kpòoló & $a d v$. last year. bara. \\
\hline lìmé & $n$. people. mutane. \\
\hline
\end{tabular}

\begin{tabular}{|c|c|}
\hline mínná & $n$. house gida. \\
\hline mínná gùp & n. palace. gidan sarki \\
\hline mínná kə̀nímmà & $n$. oracle. \\
\hline mòddồ & $n$. millet. gero. \\
\hline mòllò & $n$. brother. Kane. \\
\hline mòllà & $n$. sister. mòllà nònnó \\
\hline mòlón & $n$. relative dangi. \\
\hline \multirow[t]{2}{*}{ mòn tùyànò } & n. and adj. drunk. $b u$ \\
\hline & xilled me'. \\
\hline mòr & n. oil. mai. \\
\hline mòr tàngà & $n$. butter. man shanu. \\
\hline móotò & n. car. mota. \\
\hline múlkì & n. chieftancy. sarauta \\
\hline mùrò & v. die. mutu. \\
\hline mùshì & n. jealousy. kishi. \\
\hline
\end{tabular}




\author{
nàa n. elephant. giwa. \\ nàa prt. used to form numerals. nàa tààt thirty talatin \\ nàa fúwàt fifty hamsin. \\ náanì $\quad n$. thunder. hadari. \\ nèsshò $v$. rest. huta. \\ nèy $n$. fight. fada. \\ nìmmì $a d v$. near. kusa. ?à nìmmìm not near ba kusa ba.
}

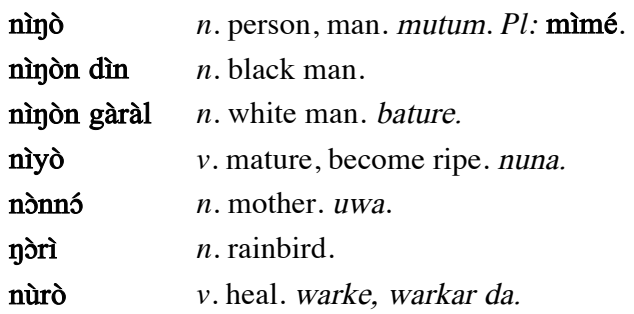

PO

Póyró $n$. bottle. kwlaba.

?ว̀ppò v. dig. haka.
Pòppò dùwò $n$. funeral. jana?iza. Lit: 'digging grave' . Tòshò v. swell. kumbura.

$\begin{array}{ll}\text { pámmò } & n . \text { ordeal. } \\ \text { pàrò } & v . \text { herd (animals). } \\ \text { pàttò } & v \text {. give birth. haifa. } \\ \text { pə̀ək } & n . \text { lungs. huhu. } \\ \text { pə̀əmún } & n . \text { female, wife mace. } P l \text { : shìrìp. } \\ \text { pə̀llı̀w } & n u m . \text { two. biyu. } \\ \text { pè } & a d j . \text { white. fari. } \\ \text { péèn } & n . \text { seed. } \\ \text { péeròw } & n u m . \text { four. hudu. }\end{array}$

péeròw num. four. hudu.

$\mathrm{R}$

$\begin{array}{ll}\text { ràagúmì } & n . \text { camel. rakumi. } \\ \text { rèeké } & n \text {. sugar cane. } \text { kara. } \\ \text { rêw } & n \text {. python. mesa. See: kúrét, shàmmàm. } \\ \text { ráwák } & n \text {. baboon. gwaggon biri. } \\ \text { rè } & v . \text { enter. shiga. }\end{array}$

réy $\quad n$. life. rai.

ríibà $n$. profit. riba.

ríijà $n$. well. rijiya.

ríwít $n$. fire-place. wurin wuta.

ròmìsh $n$. twin. dan tagwai. 


\begin{tabular}{|c|c|}
\hline sháafî & n. religious object. abin addini. \\
\hline shèewò & $v$. plant, transplant. shuka. \\
\hline shábá & n. lie. karya. \\
\hline shàylò & n. sleep. barci. \\
\hline shàmmàm & n. viper. See: kúrét; rêw. \\
\hline shàyà & $n$. bridegroom. ango. \\
\hline shànyò & $v$. exchange goods. kaya. \\
\hline shányè & $n$. yam(s). doya. \\
\hline sháppàn & n. diarrhoea. zawo. \\
\hline shàrgey & n. weapon. makami. \\
\hline shárí & n. load. kaya. \\
\hline shàw & Guinea corn. dawa. \\
\hline sháwán & n. spirit. iska. \\
\hline shə̀əd & n. morning. safiya. \\
\hline shégní & $n$. bee. Pàn lì shégní bee-keeper dòo \\
\hline & shégní honey nùn shégní queen bee. \\
\hline shègré & $n$. the past. da. \\
\hline shégrí & n. hiccough. shakuwa. \\
\hline shékì & prep between. tsakanin. \\
\hline shèllè & $n$. vulture. ungulu. \\
\hline shèmù & $n$. poison. guba. \\
\hline shén & n. cleverness. azanci, fasaha. \\
\hline shènè & $n$. year. sehkara. \\
\hline shéwó & n. 1. fear. tsoro. 2. shame. kunya. \\
\hline shí mínná & n. wall (house). bango. \\
\hline shì6ว̀ gə̀dà & n. terrace. gandu. \\
\hline shìdə̆r & $n$. fat. fet. \\
\hline shíyá & n. loss. rashi. \\
\hline shìilà & $n$. stone, rock, hill. dutse. \\
\hline shìilà bàalà & n. escarpment. dutse. \\
\hline shíiwé & $n$. firewood. itace. \\
\hline \multirow[t]{4}{*}{ shìk } & num. hundred. dari. shìk nàa one one \\
\hline & hundred dari daya shìk pòllòw two \\
\hline & hundred dari biyu shìk dôk kàn f(ú)wàt \\
\hline & hundred and five dari da biyar. \\
\hline & $n$. body. jiki. \\
\hline
\end{tabular}

\begin{tabular}{|c|c|}
\hline shìlím & n. mongoose. tunku. \\
\hline shìm & n. drum (hour glass). kalangu. \\
\hline shìm & $n$. cough tari. \\
\hline shìrìm & n. fish. kifi. \\
\hline shìrìp & n. woman. mace. \\
\hline shíwó & n. small ant. kiyashi. \\
\hline shìwòn & n. shrew. ja6a. \\
\hline shìwrì & n. long-tail rat. Gera, kusu. See: Pàllágà. \\
\hline shíyá & n. menstruation. haila. \\
\hline shò & n. leg, foot. Kafa. \\
\hline shò & v. drink. sha. \\
\hline shók & n. hair. gashi. \\
\hline shók fôk & $n$. beard. gemu. \\
\hline shók shìk & $n$. body hair. gashin jiki. \\
\hline shòkh & $n$. hair, fur, feather. gashi. \\
\hline shòmmò & $n$. name. suna. \\
\hline shòr & n. dance. rawa. \\
\hline shóorím (bìró) & n. root. saiwa. \\
\hline shù & v. eat (tuwo). ci (tuwo). \\
\hline shúná & n. dream. mafarki. \\
\hline shúyúr & n. shinbone. sangali. \\
\hline shúró & n. laugh. dariya. \\
\hline shúrùk & n. river. kogi. \\
\hline shùjì & n. male. miji. $P l$ : kpárín \\
\hline shùkkàn kírá & n. fist. dunkulen hannu. \\
\hline shùnò & $v$. skin. fede. \\
\hline shùngù & n. cloud. gajimare, girgije. \\
\hline shúurò shàw & $n$. threshing. \\
\hline shùrmì & $n$. hare. zomo. \\
\hline shùurò & v. fry. soya, toya. \\
\hline shùuyò & $v$. cheat cuta. \\
\hline shùwè & $n$. wood. itace. \\
\hline shwè & n. bush. daji, jeji. \\
\hline shwìyàk & n. sand. yashi. \\
\hline shwíyə̀k & n. dew. raba. \\
\hline
\end{tabular}




\begin{tabular}{|c|c|}
\hline tà & prep. in, at, inside. $a$, ciki $(n)$. \\
\hline tà Páà & n. stomach. ciki. \\
\hline tà 6ə̀ək & n. armpit. hamata. \\
\hline tàbùk & n. land. Kasa. \\
\hline tà gàdə̀ & $n$. slope. gangara. \\
\hline tà gùmè & $n$. entrance hut. zaure. \\
\hline tà kòn & n. legal case. Kara. \\
\hline tà mínná(mù) & n. lineage. Lit: 'inside (our) house'. \\
\hline tà mínná(nò) & n. family. Lit: 'inside (my) house'. \\
\hline tà múu & n. unfarmed ground. Lit: 'in left place'. \\
\hline tà shúrùk & $n$. valley. kwari. \\
\hline tàa & n. shoe. takalma. \\
\hline tàará & $n$. father. $u b a$. \\
\hline tàat & num. three. uku. \\
\hline tàawa & n. tobacco. taba. \\
\hline tálí & $a d v$. far. nesa. Pà tálím not far ba nesa ba. \\
\hline tàngà & $n$. bull. là tàngà calf. \\
\hline tàvrò & $n$. west. yamma. \\
\hline téelè & $n$. worm. tsutsa. \\
\hline télày & n. cooking pot. tukunya. \\
\hline èmèl & n. shoulder. kafada. \\
\hline
\end{tabular}

\begin{tabular}{|c|c|}
\hline témmè & n. small calabash. Karamin duma. \\
\hline témmè̀ & $a d v$. right. dama. \\
\hline tèmmò & $v$. sweep, clean. share. \\
\hline téré & n. moon. wata. téré pé white moon farin wata. \\
\hline tèrèt & $n$. broom. tsintsiya. \\
\hline tìtón & inter. where? ina? \\
\hline tòbbò & $v$. sow (seeds in holes). shuka, yi binne. \\
\hline tòdókù & n. fever. zazzabi. \\
\hline t’̀k & n. mat. tabarma. \\
\hline tòlśt’̀lś & n. turkey. talotalo. \\
\hline tònnò & v. 1. spin (thread). kada. 2. weave. saka. \\
\hline tòo Párát & n. Talha gum Arabic tree. Karo. \\
\hline tว̀om & prep. in front (of). a gaba(n). \\
\hline tù66ò & $n$. rubbish heap. juji. \\
\hline túyá shìgnì & $n$. unnatural death. \\
\hline tùyò & v. kill. kashe. \\
\hline tùkkò & v. 1. tie a rope daura. 2 . knot. Kulla. \\
\hline túmmò & n. guinea-fowl. zabo. \\
\hline twàanà & $n$. silk cotton tree (red flowers). rimi. \\
\hline twòné & n. small, black ant. cinnaka. \\
\hline
\end{tabular}

Pùn n. pregnancy. ciki.

$\begin{array}{ll}\text { vànà } & n . \text { black sesame. bakin ridi. } \\ \text { vàré } & n . \text { he-goat. bunsuru. } \\ \text { vèe } & n . \text { chin. ha6a. } \\ \text { vìyè } & n . \text { cobra. gamsheka. vìyè Pèlèt spitting cobra } \\ \text { vìnò } & v . \text { throw a stone. jefa dutse. } \\ \text { vìn } & n . \text { grinding stone. dutsen nika. } \\ \text { vìyò } & \text { demon, spirit. iska, aljani. } \\ \text { vìyò } & n . \text { rainy season. damina. }\end{array}$

$\begin{array}{ll}\text { vìyè } & n . \text { souce, soupe. miya. } \\ \text { vònàk } & n . \text { song. waka. } \\ \text { vòon } & n . \text { pumpkin. kabewa. } \\ \text { vùlùk } & n . \text { cave. kogon dutse. } \\ \text { vúró } & n . \text { gun powder. albarushi. } \\ \text { vùrò } & n . \text { ashes. toka. } \\ \text { vùrùm } & n . \text { knee. gwiwa. }\end{array}$


W

$\begin{array}{ll}\text { wànyì } & n . \text { trousers. wando. } \\ \text { wàré } & n . \text { mouse. Gera. } \\ \text { wàrù } & v . \text { come. zo. } \\ \text { wàw } & n . \text { sacrifice. hadaya. } \\ \text { wè bì gùrùy } & n \text {. saddle. sirdi. } \\ \text { wè fó gùrùy } & n . \text { bit (horse). linzami. } \\ \text { wè kírá } & n . \text { bracelet. abin hannu. Lit: 'thing (of) arm'. } \\ \text { wè kúmó } & n . \text { earring. 'yan kunne. Lit: 'thing (of) ear'. } \\ \text { wè túr } & n . \text { necklace. abin wuya. Lit: 'thing of neck'. } \\ \text { wòrì } & n . \text { breast. nono. } \\ \text { wìirì } & n . \text { Indian tamarind. tsamiya. } \\ \text { wìshò } & v . \text { roast. gasa. }\end{array}$

$\begin{array}{ll}\text { wìyàk } & n . \text { bone. Kashi. } \\ \text { wòjò } & v . \text { avoid, abstain. Karauce wa. } \\ \text { wòkù } & n . \text { helmet. hular kwano. } \\ \text { wòkù } & n . \text { cap. hula. } \\ \text { wúró } & n . \text { tooth. hakori. } \\ \text { wùjìn } & n . \text { nose. hanci. } \\ \text { wùlù } & v . \text { strike fire. kunna wuta. } \\ \text { wùllò } & v . \text { bubble, ferment. ruba. } \\ \text { wùrì } & n . \text { breast. nono. } \\ \text { wùrìn } & n . \text { medicine. magani. } \\ \text { wùròk } & n . \text { pus. mugunya. }\end{array}$

Y

$\begin{array}{ll}\text { yù mə̀n } & v . \text { brew (beer). dafa. } \\ \text { yù mèrà } & v \text {. travel. yi tafiya. } \\ \text { yàamúyì } & n \text {. brother-in-law. Kane, wa. } \\ \text { yàllò } & n \text {. shouting. ihu. } \\ \text { yàmmà } & n \text {. god. Allah, Ubangiji. } \\ \text { yàrà shwè } & n \text {. hunt. farauta. } \\ \text { yèrà } & n \text {. itch. Kaikayi. } \\ \text { yèrì } & n . \text { red ant. jan kiyashi. } \\ \text { yèrò } & v \text {. bite. ciza. } \\ \text { yìbà dèet } & n \text {. flame. harshen wuta. } \\ \text { yìrà } & n . \text { reception. liyafa. }\end{array}$

$\begin{array}{ll}\text { yípó } & n \text {. evening, night, darkness. dare, duhu. } \\ \text { yìipò shìrìim } & v \text {. to fish. sunta, yi fatsa. } \\ \text { yò } & v \text {. call. kira. } \\ \text { yù } & v \text {. do, make. yi. } \\ \text { yù 6èrà } & v \text {. cut marks. shasshauta. } \\ \text { yù dìyò } & v \text {. marry. yi aure. } \\ \text { yù gùp } & v \text {. rule. yi mulki. } \\ \text { yù jírí } & v \text {. steal. sata. } \\ \text { yù nèy } & v \text {. fight. yi fada. } \\ \text { yù shìlìp } & v \text {. suffer. sha wahala. } \\ \text { yùylà } & n \text {. medium size calabash. Kwarya. }\end{array}$




\section{ENGLISH-KUSHI}

A

\begin{tabular}{|c|c|}
\hline \multirow{3}{*}{$\begin{array}{l}\text { above } \\
\text { abstain } \\
\text { accusation }\end{array}$} & prep. dày (2). \\
\hline & $v$. wòjò. \\
\hline & n. gùlmà. \\
\hline across & prep. gbònnà. \\
\hline age-mate & $n$. yèerè. \\
\hline ancestor & $n$. jìijí (2). \\
\hline anklet & n. gbárlàm. \\
\hline ant & n. dòlòní; \\
\hline & n. fિy̋́; \\
\hline & n. shíwó; \\
\hline & $n$. twòné; \\
\hline & $n$. yèrì; \\
\hline & n. 'áyúkh. \\
\hline
\end{tabular}

\begin{tabular}{|c|c|}
\hline antelope & $n$. bànà. \\
\hline ant-hill & $n$. màmmà. \\
\hline anus & $n$. fô gùn; \\
\hline & $n$. gùn. \\
\hline aridity & $n$. jènè. \\
\hline arm & n. kírá. \\
\hline armpit & n. tà 6ə̀ək. \\
\hline arrow & $n$. fî̀k. \\
\hline ashes & $n$. vùrò. \\
\hline at & prep. tà. \\
\hline avoid & v. wòjò. \\
\hline axe & $n$. gbàamé. \\
\hline
\end{tabular}

B

\begin{tabular}{|c|c|c|c|}
\hline baboon & n. rówák. & blacksmith & n. Pàn kùwà. \\
\hline back & $n$. bì (1). & blanket & n. gódó. \\
\hline bag & n. lúrú. & blind & $n$. fùgùm. \\
\hline bambara nut & $n$. dùurò. & body & $n$. shìk. \\
\hline baobab & n. Gánnì. & boil & v. dûmmò. \\
\hline \multirow[t]{3}{*}{ basket } & n. gánà; & bone & $n$. wìyàk. \\
\hline & $n$. jàrà; & border & $n$. kə̀rə̀k. \\
\hline & $n$. jùnroy. & bottle & n. 'วิүró. \\
\hline bean $(s)$ & $n$. jəેə. & bracelet & $n$. wè kírá. \\
\hline beard & n. shók fôk. & branch & n. kírá bìró. \\
\hline beat & v. Gìrò. & breast & $n$. wə̀rì; \\
\hline bed & $n$. jìrà. & & $n$. wùrì. \\
\hline bee & $n$. shégní. & brew & $v$. yù mə̀n. \\
\hline beer & $n$. mə̀n. & bridegroom & $n$. shànà. \\
\hline beget & $v \cdot$ pàttò. & broom & $n$. tèrèt. \\
\hline behind & prep. bì (2). & brother & n. mòllò. \\
\hline bell & n. káráráwá. & bubble & $v$. wùllò. \\
\hline bellows & $n$. fùgrò. & bucket & $n$. bùkwátì. \\
\hline belly & n. gònว̀. & build & $v$. dìgù. \\
\hline between & prep. shékì. & bull & $n$. tàygà. \\
\hline big & adj. gbé. & bundle & v. dáwán. \\
\hline bird & n. Pàdéewè. & bush & $n$. shwè. \\
\hline bit & $n$. wè fó gùrùy. & butcher & n. Pàn páawà \\
\hline bite & $v \cdot$ yèrò. & butter & $n$. mòr tàngà. \\
\hline black & adj. dìn ${ }_{2}$. & butterfly & $n$. lìlámdè. \\
\hline
\end{tabular}




\begin{tabular}{|c|c|}
\hline calabash & $n$. gùráy; \\
\hline call & v. yò. \\
\hline camel & $n$. ràagúmì. \\
\hline cane (sugar) & $n$. rèeké. \\
\hline cap & $n$. wòkù. \\
\hline car & n. móotò. \\
\hline carve & v. gbàayò. \\
\hline case & $n$. tà kòn. \\
\hline caterpillar & $n$. jéné. \\
\hline cave & $n$. vùlùk. \\
\hline chair & $n$. jùk $\mathbf{k}_{2}$. \\
\hline charcoal & n. dìmlìm (1) \\
\hline charm & n. láayá. \\
\hline cheat & $v$. shùuyò. \\
\hline cheek & $n$. jáylùm. \\
\hline chest & $n$. gbìilì. \\
\hline chicken & $n$. féè. \\
\hline chief & $n$. gùp. \\
\hline chieftancy & n. múlkì. \\
\hline child & $n$. là. \\
\hline chin & $n$. vèe. \\
\hline circumcision & n. kàr kúwò. \\
\hline claw & n. kòdô. \\
\hline clay & n. dગ̂ybó. \\
\hline clean & $v$. tèmmò. \\
\hline
\end{tabular}

\begin{tabular}{|c|c|}
\hline cleverness & $n$. shén. \\
\hline climb up & v. Pàmmò (1). \\
\hline clothes & $n$. lànnà. \\
\hline cloud & $n$. shùngù. \\
\hline cobra & $n$. vìyè. \\
\hline cock & $n$. gòkh. \\
\hline cocoyam & $n$. gwàaja. \\
\hline come & $v$. wàrù. \\
\hline come out & $v$. pìrù. \\
\hline cook & v. dînò. \\
\hline cool & $v$. dûulò. \\
\hline cornstalk & $n$. 'ìshì shwè. \\
\hline corpse & n. kúmá. \\
\hline cotton & $n$. lèeláw. \\
\hline $\operatorname{cough}_{2}$ & n. shìm. \\
\hline courage & n. Gán fôk. \\
\hline cow & $n$. khèwnè. \\
\hline cowrie & $n$. kènè dîyò. \\
\hline cowry & $n$. khùrùwò. \\
\hline crab & $n$. jènyè. \\
\hline cricket & $n$. jwàlé. \\
\hline crocodile & $n$. kèrèm. \\
\hline crow & $n$. gàakh. \\
\hline curse & n. kùwák. \\
\hline cutlery & $n$. jáwít. \\
\hline
\end{tabular}

D

\begin{tabular}{|c|c|c|c|}
\hline dance & $n$. shòr. & do & $v \cdot$ yù. \\
\hline dancer & n. Pàn shòr. & $\operatorname{dog}$ & n. 6ày. \\
\hline darkness & n. yípó. & donkey & $n$. gìrbì. \\
\hline date & n. gòylày. & door & n. dán. \\
\hline daughter & $n$. là shìrìp. & doorway & n. fô dán. \\
\hline daughter-in-law; son-in-law & $n$. gbàrìm. & doubt & $n$. kùmùm. \\
\hline dawn & n. kíríyípó. & dream & n. shúná. \\
\hline daylight & $n$. kà dàrày. & drink & $v$. shò. \\
\hline debt & n. kúwó. & drum $_{1}$ & n. gàngày; \\
\hline demon & $n$. vìyò. & & $n$. shìm. \\
\hline desease & n. pírá. & drummer & n. Pàn gàngày. \\
\hline desire & n. léyá. & drunk & n. and adj. mòn tùyànò. \\
\hline dew & n. shwíyə̀k. & dry & adj. 'íshàn. \\
\hline diarrhoea & n. sháppàn. & dry up & $v$. Pìshò. \\
\hline die & $n$. mùrò. & dryness & $n$. jènè. \\
\hline dig & v. 1ร́ppò. & duck & n. Pàgwàgwá. \\
\hline divorce & n. 6wàarò dîyò. & dysentery & n. kúnná. \\
\hline
\end{tabular}

Linguistics Discovery 15.1:85-106 
E

\begin{tabular}{|c|c|c|c|}
\hline ear & n. kúmó. & enemy & $n$. Pàn gèy. \\
\hline earring & n. wè kúmó. & enter & $v \cdot$ rè. \\
\hline east & n. 6òolì. & escape & $n$. fôorà kúnò. \\
\hline \multirow[t]{2}{*}{ eat } & $v$. shù. & escarpment & $n$. shìilà Gàalà. \\
\hline & v. ใàddô. & evening & n. kíríyípó; \\
\hline eight & num. pìdìdôw. & & n. yípó. \\
\hline elbow & $n$. gùngúl. & exchange & v. shànyò. \\
\hline elder sisters of mother & n. nònnó-nònnó, see: nònnó. & eye & n. Pàdô (1). \\
\hline elephant & $n$. jùk & & \\
\hline & $n$. nàa. & & \\
\hline
\end{tabular}

F

\begin{tabular}{|c|c|c|c|}
\hline face & n. Pàdô (2). & fist & $n$. shùkkàn kírá. \\
\hline family & n. tà mínná(nò). & five & num. fúwàt. \\
\hline far & $a d v$. tálí. & flail & n. Gìr(î) shàw. \\
\hline farm & n. dذ̀y. & flame & $n$. yìbà dèet. \\
\hline fat & $n$. shìdəิr. & flea & $n$. gàarì. \\
\hline father & $n$. tàará. & flesh & n. lذે. \\
\hline fear & $n$. shéwó (1). & flour & $n$. lùmmò. \\
\hline feather & $n$. shว̀kh. & flower & $n$. bìlìr. \\
\hline female & $n$. pə̀əmún. & fly & n. dùyó; \\
\hline fence & n. dàngà; & & $n$. fuumò̀; \\
\hline ferment & $v$. wùllò. & foam & n. fúvùl. \\
\hline festival & n. dàbán. & food & $n$. gwàn. \\
\hline fetish & $n$. kə̀nímmà. & foot & n. shò. \\
\hline fever & $n$. t’̀dókù. & footprint & $n$. bì shò. \\
\hline field & n. d’̀y. & forehead & n. fગิorò. \\
\hline \multirow[t]{2}{*}{ fight } & $n$. nèy; & forest & n. dìl bìró. \\
\hline & $v \cdot$ yù nèy. & forge & $v$. kùwà. \\
\hline \multirow[t]{2}{*}{ filter } & $n$. jòd; & four & num. péeròw. \\
\hline & $v$. kòshò. & francolin & $n$. Pàntórò. \\
\hline finger & $n$. fô kírá. & friend & $n$. jèrè. \\
\hline fire & $n$. dèet. & frog & n. dànyùkh. \\
\hline fire-place & $n$. ríwít. & fry & $v$. shùurò. \\
\hline firewood & n. shíiwé. & funeral & n. '’̀ppò dùwò. \\
\hline fish & $n$. shìrìm; & fur & $n$. shòkh. \\
\hline
\end{tabular}




\begin{tabular}{|c|c|}
\hline garden & $n$. làmbú. \\
\hline gazelle & n. 'ádáy. \\
\hline gecko & n. Pàntúgó. \\
\hline ghost & n. dùwò. \\
\hline give birth & $v \cdot$ pàttò. \\
\hline go out & $v \cdot$ pèrò. \\
\hline goat & $n$. vàré. \\
\hline god & $n$. yàmmà. \\
\hline goodness & n. gbán. \\
\hline grain & $n$. 'áeshàw \\
\hline granary & n. kán. \\
\hline grandfather & n. jìijí (1 \\
\hline
\end{tabular}

\begin{tabular}{|c|c|}
\hline grandmother & $n$. gàayá. \\
\hline grasshopper & $n$. gòlòm. \\
\hline grave & $n$. dùwò. \\
\hline grind & $v$. Pìshò. \\
\hline grinding stone & $n$. vìn. \\
\hline ground & n. tà 6ùk, kù 6ùk \\
\hline groundnut & $n$. dùwàaynò. \\
\hline gruel & $n$. kháa. \\
\hline guest & $n$. bìnnò. \\
\hline guinea-fowl & n. túmmò. \\
\hline gun & $n$. bìndìgà. \\
\hline
\end{tabular}

$\mathrm{H}$

\begin{tabular}{|c|c|}
\hline hail & $n$. Pàdéelè. \\
\hline \multirow[t]{2}{*}{ hair } & $n$. shók; \\
\hline & $n$. shòkh. \\
\hline hand & n. kírá. \\
\hline hare & $n$. shùrmì. \\
\hline \multirow[t]{2}{*}{ harvest } & n. káshò; \\
\hline & $v$. kàshò. \\
\hline hawk; eagle & $n$. gètè. \\
\hline head & $n$. khù. \\
\hline headpad & n. gbóshì. \\
\hline heal & $v$. nùrò. \\
\hline healer & $n$. Pàn wùrìn. \\
\hline heart & $n$. fo shə̀ərì. \\
\hline heaven & $n$. kù dày (2) \\
\hline hedgehog & n. Pàkùmóm. \\
\hline heel & $n$. bìirìm. \\
\hline helmet & $n$. wòkù. \\
\hline herd & $v \cdot$ pàrò. \\
\hline
\end{tabular}

\begin{tabular}{|c|c|}
\hline here & $a d v$. fôtè. \\
\hline hiccough & n. shégrí. \\
\hline hill & n. shìilà. \\
\hline hoe & $\begin{array}{l}n . \text { dúmmò; } \\
v . \text { káyò. }\end{array}$ \\
\hline hollow out & v. gbáayò. \\
\hline hook & $n$. pìl. \\
\hline horn & n. kpàa; \\
\hline & n. Pàdúnúnò. \\
\hline horse & $n$. gùrùy. \\
\hline house & n. mínná. \\
\hline hundred & num. shìk. \\
\hline hunger & n. kúyám. \\
\hline hunt & $n$. yàrà shwè. \\
\hline hunter & $n$. Pàn tà shwè \\
\hline husband & n. mánná. \\
\hline hut & $n$. gùmè. \\
\hline hyena & $n$. dà66à. \\
\hline
\end{tabular}

I

in prep. tà.

itch $n$. yèrà.

$\mathrm{J}$
jaw $n$.ján.
jealousy $n$. mùshì. 


$$
\begin{array}{ll}
\text { kill } & v . \text { tùyò. } \\
\text { king } & n . \text { gùp. } \\
\text { knee } & n . \text { vùrùm. }
\end{array}
$$

\begin{tabular}{|c|c|}
\hline lame & $n$. gùngù. \\
\hline land & n. tà 6ùk, kù 6ùk. \\
\hline language & $n$. fિk (2). \\
\hline last year & $a d v$. mìllán kpòols. \\
\hline laterite & n. dìn. \\
\hline laugh & n. shúró. \\
\hline lazy & n. and adj. kùulè. \\
\hline leaf & n. Pàlàw. \\
\hline leatherworker & n. 'an límmò láyát. \\
\hline left & $a d v$. jìyaw. \\
\hline leg & n. shò. \\
\hline lend & v. kùwò. \\
\hline leopard & n. jàyàní. \\
\hline lie & n. shábá. \\
\hline
\end{tabular}

$$
\begin{array}{ll}
\text { knife } & n . \text { jáwít. } \\
\text { knot } & v \text {. tùkkò (2). }
\end{array}
$$

\begin{tabular}{|c|c|}
\hline life & $n$. réy. \\
\hline lightning & $n$. màlyò. \\
\hline lineage & n. tà mínná(mù). \\
\hline lion & $n$. kàmmà. \\
\hline like & $v$. lèwò. \\
\hline liver & n. fô lùwàk. \\
\hline lizard & $n$. kèlà; \\
\hline & n. Pàntúgó. \\
\hline load & n. shárí. \\
\hline locust & n. jórró. \\
\hline loom & $n$. jàmmó. \\
\hline loss & n. shíyá. \\
\hline love & $v$. lèwò. \\
\hline lungs & $n$. pə̀ək. \\
\hline
\end{tabular}

\begin{tabular}{|c|c|}
\hline maize & $n$. kòomò. \\
\hline make & $v \cdot$ yù. \\
\hline malaria & $n$. filéríyà. \\
\hline male & $n$. shùjì; \\
\hline & n. shújí. \\
\hline mantis & n. Pàkúlà. \\
\hline many & $a d v$. mè Pàn. \\
\hline mare & $n$. gùrùn. \\
\hline market & n. káasùwà. \\
\hline marriage & n. dîyò. \\
\hline marry & $v$. yù dîyò. \\
\hline mat & $n$. tòk. \\
\hline mature & $v$. nìyò. \\
\hline measure & $v$. kèrò. \\
\hline meat & n. lòे. \\
\hline medicine & $n$. wùrìn. \\
\hline
\end{tabular}

$\mathrm{L}$

\begin{tabular}{|c|c|}
\hline menstruation & $n$. shíyá. \\
\hline milk & $n$. dòo wùrì. \\
\hline millet & $n$. mòddô. \\
\hline molar & $n$. gə̀rùm. \\
\hline money & n. kónó. \\
\hline mongoose & $n$. shìlím. \\
\hline monkey & $n$. jìró. \\
\hline moon & $n$. téré. \\
\hline morning & $n$. shə̀əd. \\
\hline mortar & n. dîikó. \\
\hline mosquito & $n$. pèrèt. \\
\hline mother & n. nònnó. \\
\hline mother's sister & n. mòllà nònnó, see: mòllà. \\
\hline mouse & $n$. wàré. \\
\hline mouth & $n$. fok (1). \\
\hline murderer & $n$. Pàn tùy nìnyò. \\
\hline
\end{tabular}

M 
N

$\begin{array}{ll}\text { name } & n . \text { shòmmò. } \\ \text { natron } & n . \text { bàkà. } \\ \text { navel } & n . \text { kúmmí. } \\ \text { near } & a d v \text {. nìmmì. } \\ \text { neck } & n \cdot \text { gbón. } \\ \text { necklace } & n \cdot \text { wè túr. }\end{array}$

oil n. mòr.

okra n. Pá6ùrùl.

on prep. kù.

one num. dらk.

onion $n$. Tàrùm.

oracle $n$. mínná kə̀nímmà.

$\begin{array}{ll}\text { needle } & n . \text { Pàlúurà. } \\ \text { night } & n . \text { yípó. } \\ \text { nine } & n u m . \text { foyèràw. } \\ \text { noon } & n . \text { kà dàrày. } \\ \text { north } & n . \text { làdî. } \\ \text { nose } & n . \text { wùjìn. }\end{array}$

$\mathrm{O}$
ordeal
n. pámmò.
ornament $n$.gbálán.
orphan
n. làkà.
outside $a d v$. péewé.
owl
$n$. mìigù.

$\mathrm{P}$

\begin{tabular}{|c|c|}
\hline palace & n. mínná gùp. \\
\hline past & $n$. shègré. \\
\hline pawpaw & $n$. góndà. \\
\hline pea & $n$. 'árí. \\
\hline peasant & n. Pàn shìráw. \\
\hline penis & $n$. kùwò. \\
\hline people & $n$. mìmé. \\
\hline pepper & $n$. Pànshìndáw. \\
\hline person & $n$. nìyò. \\
\hline pestle & n. gbánlày. \\
\hline pig & $n$. Pàládè. \\
\hline pipe & n. dôorò. \\
\hline plant & $v$. shèewò. \\
\hline pluck out & v. fિddఏ. \\
\hline poison & $n$. shèmù. \\
\hline poor & $n$. kènnì. \\
\hline
\end{tabular}

$\begin{array}{ll}\text { porcupine } & n . \text { jêy. } \\ \text { pot } & n . \text { dìgré; } \\ & n \text {. díyè; } \\ & n \text {. là tálày; } \\ & n \text {. télày. } \\ & n . \text { làn díyè. } \\ \text { potter } & v . \text { dìishò. } \\ \text { pound } & n . \text { vúró. } \\ \text { powder } & n . \text { lùn. } \\ \text { pregnancy } & n . \text { làn gùnùl. } \\ \text { priest } & n . \text { ríibà. } \\ \text { profit } & n . \text { vòon. } \\ \text { pumpkin } & n . \text { fòoró. } \\ \text { punishment } & n . \text { wùròk. } \\ \text { pus } & n . \text { lí. } \\ \text { put } & n . \text { rêw. } \\ \text { python } & \end{array}$




\begin{tabular}{|c|c|}
\hline rainbird & n. yòrì. \\
\hline rainbow & $n$. màlàc. \\
\hline rainmaker & n. Pàn kə̀nímmà. \\
\hline ram & $n$. gàlmà. \\
\hline rat & n. dàmàl; \\
\hline & $n$. gàrìnjìn; \\
\hline & $n$. shìwrì; \\
\hline & n. Pàllágà; \\
\hline & n. Pàyùkh. \\
\hline rattle & n. Gwáré. \\
\hline reception & $n$. yìirà. \\
\hline red & adj. gàyàl. \\
\hline relative & $n$. mòlón. \\
\hline rest & $v$. nèsshò. \\
\hline ribs & $n$. jèrà gònò. \\
\hline rice & n. gàpà. \\
\hline
\end{tabular}

\begin{tabular}{|c|c|}
\hline rich & $n$. jìkò. \\
\hline ride horse & v. Pàmmò (2). \\
\hline right & $a d v$. témmè \\
\hline ring & $n$. 'èm. \\
\hline river & $n$. shúrùk. \\
\hline riverbank & $n$. fô shúrùk. \\
\hline road & n. kóyó. \\
\hline roast & $v$. wìshò. \\
\hline rock & n. shìilà. \\
\hline roof & $n$. kù mínná. \\
\hline room & $n$. gùmè. \\
\hline root & n. shóorím (bìró). \\
\hline rope & n. fúulá. \\
\hline ruin & n. dòybó. \\
\hline rul & $v$. yù gùp. \\
\hline nate & $v$. làllò. \\
\hline
\end{tabular}




\begin{tabular}{|c|c|}
\hline sacrifice & $n$. wàw. \\
\hline saddle & $n$. wè bì gùrùn. \\
\hline salt & n. Pànjà. \\
\hline sand & $n$. lùmmò bùk; \\
\hline & $n$. shwiyàk. \\
\hline scar & $n$. jwál fíră. \\
\hline scorpion & n. 'ádénré. \\
\hline seed & $n$. péèn. \\
\hline senior & adj. gbé. \\
\hline servant & n. là mínná. \\
\hline sesame & $n$. khèeyàw; \\
\hline & n. ládú; \\
\hline & $n$. vànà; \\
\hline & n. ’árkyaw. \\
\hline seven & num. fârlów. \\
\hline sew & $v$. tònnò (1). \\
\hline shade & $n$. lùgnì. \\
\hline shame & $n$. shéwó (2). \\
\hline sheep & $n$. dìnyì. \\
\hline shell & n. gગેylગ̀. \\
\hline shield & $n$. jèdè. \\
\hline shinbone & n. shúyúr. \\
\hline shoe & $n$. tàa. \\
\hline shoot & $v$. bèe. \\
\hline shoulder & $n$. tèmèl. \\
\hline shouting & $n$. yàllò. \\
\hline shrew & $n$. shìwòn. \\
\hline sickle & $n$. fિdə́mlì. \\
\hline side & $n$. yèlà. \\
\hline sinew & $n$. dèy. \\
\hline singer & $n$. Pàn vònàk. \\
\hline sister & $n$. mòllà. \\
\hline $\operatorname{six}$ & num. fâràgbànày \\
\hline skin & $v$. shùnò. \\
\hline skin (body) & $n$. láyát (shìk). \\
\hline skull & $n$. màylày. \\
\hline sky & $n$. kù dày (1). \\
\hline sleep & $n$. shàylò. \\
\hline slope & $n$. tà gàdə̀. \\
\hline smoke & $n$. dùnò. \\
\hline
\end{tabular}

\begin{tabular}{|c|c|}
\hline snake & $\begin{array}{l}n . \text { kpèyù; } \\
n . \text { kúrét. }\end{array}$ \\
\hline snare & $v$. jìwò. \\
\hline soil & $n$. bùk. \\
\hline son & $n$. là. \\
\hline song & $n$. vònàk. \\
\hline soot & n. dìmlìm (2). \\
\hline sorcerer & n. Pàn shòo. \\
\hline sore & n. pírá. \\
\hline souce & $n$. vìyè. \\
\hline soup & $n$. vìyè. \\
\hline south & n. dày (1). \\
\hline sow & $\begin{array}{l}v . \text { kàawò; } \\
v . \text { tòbbò. }\end{array}$ \\
\hline spear & $n$. kàsh. \\
\hline spider & n. láalá. \\
\hline spin & $v$. tònnò (1). \\
\hline spirit & n. dòodó; \\
\hline & $n$. jìrìm; \\
\hline & n. sháwán; \\
\hline & $n$. vìyò. \\
\hline spoon & $n$. jáwít. \\
\hline squirrel & $n$. júr. \\
\hline stallion & $n$. gùrùn. \\
\hline star & $n$. Pày gèerù téré; \\
\hline & n. Pàrshàl. \\
\hline steal & $v \cdot$ yù yírí. \\
\hline stomach & $n$. tà 'áà. \\
\hline stone & $n$. shìilà. \\
\hline storm & $n$. pìrìt dòo. \\
\hline stranger & n. bìnnò. \\
\hline straw & $n$. jùjákh. \\
\hline strength & n. Gáy. \\
\hline strip off & v. gbáarò. \\
\hline suffer & $v \cdot$ yù shìlìp. \\
\hline sun & n. dàràn. \\
\hline sunshine & $n$. dàrày dén. \\
\hline sweep & $v$. témmò. \\
\hline swell & v. Pòshò. \\
\hline sword & $n$. Pàngárùm. \\
\hline
\end{tabular}




\begin{tabular}{|c|c|}
\hline tail & $n$. gìrìr. \\
\hline tamarind & $n$. wìirì. \\
\hline $\tan$ & $v$. kpàanò. \\
\hline tanner & n. Pàn kpáanì. \\
\hline taro & n. gwàaja. \\
\hline $\operatorname{tax}$ & n. gàndù. \\
\hline ten & num. kpòmù. \\
\hline termite & $n$. khàyukh. \\
\hline terrace & n. shìbò gàdə̀. \\
\hline testicles & n. Pàr shíilì. \\
\hline there & $a d v$. fô tèerè; \\
\hline & $a d v$. fôshénnì. \\
\hline thief & n. jírí. \\
\hline thigh & $n$. fînnè. \\
\hline thirst & $n$. 'ìshì fôk. \\
\hline thorn & n. Pàràt. \\
\hline thousand & num. dùbù. \\
\hline three & num. tàat. \\
\hline threshing & $n$. shúurò shàw \\
\hline throat & $n$. gòoròk. \\
\hline thunder & n. náanì. \\
\hline t: & $v$. tùkkò (1). \\
\hline me & n. dímím. \\
\hline
\end{tabular}

uncle $n$. fonnò.

under prep. gùm.

\begin{tabular}{|c|c|}
\hline tiredness & n. Gálá. \\
\hline to fish & $v \cdot$ yìipò shìrìim \\
\hline toad & $n$. dànyùkh. \\
\hline tobacco & $n$. tàawa. \\
\hline today & $n$. bwòy. \\
\hline \multirow[t]{2}{*}{ tomato } & $n$. kùurò dòo; \\
\hline & $n$. kùurò. \\
\hline tomorrow & n. dwèy. \\
\hline tongue & n. dák. \\
\hline tooth & n. wúró. \\
\hline track & n. khóyó. \\
\hline transplant & $v$. shèewò. \\
\hline trap & $n$. bàrà. \\
\hline \multirow[t]{2}{*}{ travel } & $n$. mèrà; \\
\hline & $v$. yù mèrà. \\
\hline tree & $n$. bìró. \\
\hline trousers & n. wànyì. \\
\hline trumpet & $n$. jèshílè. \\
\hline trunk & n. 6òy bìró. \\
\hline turkey & n. tòlótว̀ló. \\
\hline twin & $n$. ròmìsh. \\
\hline two & num. pàllòw. \\
\hline
\end{tabular}

$\mathrm{U}$ $\begin{array}{ll}\text { underworld } & n . \text { bwèyù. } \\ \text { unripe } & a d j . \text { Pàníyùm. }\end{array}$

V

$\begin{array}{ll}\text { viper } & n . \text { shàmmàm. } \\ \text { voice } & n . \text { 'élé. } \\ \text { vulture } & n . \text { shèllè. }\end{array}$


W

\begin{tabular}{|c|c|}
\hline wall & n. shí mínná. \\
\hline want & $v$. lèwò. \\
\hline war & n. gèy. \\
\hline warrior & n. Pàn tòom. \\
\hline wash & v. fûddô. \\
\hline \multirow{2}{*}{ wasp } & $n . \mathbf{j} \grave{\mathbf{s k}}$ \\
\hline & $n$. méemé. \\
\hline water & n. dòo. \\
\hline weapon & $n$. shàrgey. \\
\hline weave & $v$. tònnò (2). \\
\hline weaver & n. Pàn láddò. \\
\hline weed & v. fòddうิ. \\
\hline well & n. ríijà. \\
\hline west & $n$. tàvrò. \\
\hline & adj. dúulàn. \\
\hline when? & inter. dím \\
\hline
\end{tabular}

where? inter. tìtón.

white adj. pè.

widow $n$. kòlò.

wife $n$. pə̀əmún.

wind $n$. pìrìt.

wing n. bàakh.

wing(s) n. 6ə̀ək.

witch $n$. ?àn wòlò.

woman $n$. shìrìp.

wood $n$. shùwè.

world $n$. kù péewè.

worm $n$. jìwúr;

$n$. téelè.

worship $v$. fôdûlò.

wound n.pírá;

$v$. khà.

X

xylophon $n$. gájágí.

Y

yam(s) n. shányè.

yesterday $n$. kpòoló.

year $n$. shènè. 